EPJ Web of Conferences 116, 10002 (2016)

DOI: $10.1051 /$ epjconf/201611610002

(C) Owned by the authors, published by EDP Sciences, 2016

\title{
Capabilities of IceCube's gamma-ray, optical and X-ray follow-up programs
}

\author{
Thomas Kintscher ${ }^{\mathrm{a}}$ for the IceCube Collaboration \\ Deutsches Elektronen-Synchrotron (DESY), Platanenallee 6, 15738 Zeuthen, Germany
}

\begin{abstract}
The IceCube neutrino observatory is a $1 \mathrm{~km}^{3}$ detector for Cherenkov light in the ice at the South Pole. Although the presence of a diffuse astrophysical neutrino flux has been confirmed, its origin has yet to be resolved. Given the current constraints on continuous point source searches, transient and variable objects emerge as promising, detectable source candidates. IceCube boosts the sensitivity to these types of sources by alerting third-party observatories of neutrino events clustered in direction and time. This paper will showcase several neutrino-triggered multi-messenger programs in IceCube along with their results and prospects.
\end{abstract}

\section{Introduction}

Understanding the origin of cosmic rays has been a long standing goal in astrophysics. Multimessenger observations, here using neutrinos and cosmic rays, can provide a viable tool in locating and understanding cosmic acceleration sites. Hadronic interactions producing cosmic rays can also produce high-energetic neutrinos [1], which reach earth unabsorbed and undeflected and whose arrival directions point back to their origin likely coinciding with the sources of cosmic rays.

The IceCube experiment has shown evidence for the existence of a diffuse astrophysical neutrino flux [2], the sources of which have yet to be identified. In addition to the search for steady sources [3], transient and variable objects present further possible source candidates. Simultaneous observations of neutrinos and photons during their various states of activity can improve the sensitivity to these sources and yield a more complete picture of their acceleration mechanisms.

In gamma rays the availability of observations is limited to certain points of time in the past due to the field of view of optical, Cherenkov and X-ray telescopes, covering typically just several square degrees. In contrast, neutrino telescopes are able to observe the full sky around the clock with a duty cycle close to $100 \%$. Using them as a trigger for optical, gamma-ray and X-ray telescopes can therefore yield more observations in e.g. gamma rays and correlated neutrinos.

So called Neutrino triggered Target of Opportunity (NToO) programs need to cover several order of magnitude w.r.t. the time-scale on which sources' activities vary: They range from milliseconds (e.g. fast radio bursts) over days to years in the case of active galactic nuclei (AGN) [4]. Thus, the

\footnotetext{
a e-mail: thomas.kintscher@desy.de
}

This is an Open Access article distributed under the terms of the Creative Commons Attribution License 4.0, which permits unrestricted use, distribution, and reproduction in any medium, provided the original work is properly cited. 
analysis chains are highly automated and alerts of neutrino flares can be generated without human intervention.

Currently, IceCube operates two of these NToO programs: the gamma-ray follow-up (GFU) and the optical and X-ray follow-up (OFU, XFU). After the description of the detector in Sect. 2, the operation of both programs is explained in Sect. 3. Section 4 closes with a summary.

\section{The IceCube detector}

The IceCube neutrino observatory is a cubic-kilometer detector for Cherenkov light, installed in the ice at the geographic South Pole [5]. The construction of the detector, i.e. deployment of its 5160 optical modules, was completed in 2010. Of the possible kinds of interactions, the selection of muon tracks from charged-current muon neutrino interactions in the detector or nearby bedrock is of particular interest to this analysis. The track-like signature of the muon can be used to reconstruct the direction of the muon and corresponding neutrino with a median precision of one degree.

The muon filter at the South Pole selects these events with a rate of $40 \mathrm{~Hz}$. In order to suppress the background from atmospheric muons, the Online Level 2 filter uses likelihood reconstructions, which take into account photon propagation and the ice properties. As computing power at the South Pole is limited, computationally demanding reconstructions are only applied to events passing further cuts. After cuts on the likelihood fit quality, the event rate is reduced to $5 \mathrm{~Hz}$. Afterwards, multivariate classifiers are employed to improve the neutrino purity to $90 \%$ at an event rate of $5 \mathrm{mHz}$.

\section{Follow-up programs}

As mentioned before, two follow-up programs are currently in operation. In the past, their respective event selection and clustering analysis was run at the South Pole and only the most significant clusters triggering an alert were sent to the North through a low-latency satellite link. Such an alert then triggered an automatically generated email to the follow-up observatories.

In order to improve the flexibility of the system and facilitate maintenance the design is about to be changed: Information (e.g. direction, energy, fit quality and uncertainty estimates) about every wellreconstructed, track-like event passing a common event selection will be sent immediately through the Iridium satellite network. After a median delay of 22 seconds after the neutrino interaction, events are received in the North and multiple analyses with different physics goals run on the events from the stream.

\subsection{Gamma-ray follow-up}

Catching flares from known, variable sources such as AGN is the aim of the so-called gamma-ray follow-up program. Upon observing an event in IceCube, a time-dependent point source analysis is run on a list of pre-agreed upon source candidates. This list is comprised of AGN from the Fermi catalog [6] in the northern hemisphere, which have shown variable behavior in gamma rays in the past and are visible (in terms of direction and redshift) to the respective follow-up telescope. An unbinned maximum likelihood analysis considering the quality of the angular reconstruction as well as the energy spectrum of the events fits two quantities: The spectral index of the events and the most likely number of signal events among the events in the time window.

The significance of the result is determined using a likelihood ratio test of the likelihood given the best fitted spectral index and number of signal events versus the likelihood assuming only background events. By testing multiple, increasing time windows starting from the latest (triggering) event and reaching up to three weeks in the past, the most likely temporal extent of a cluster of neutrinos can be determined. 


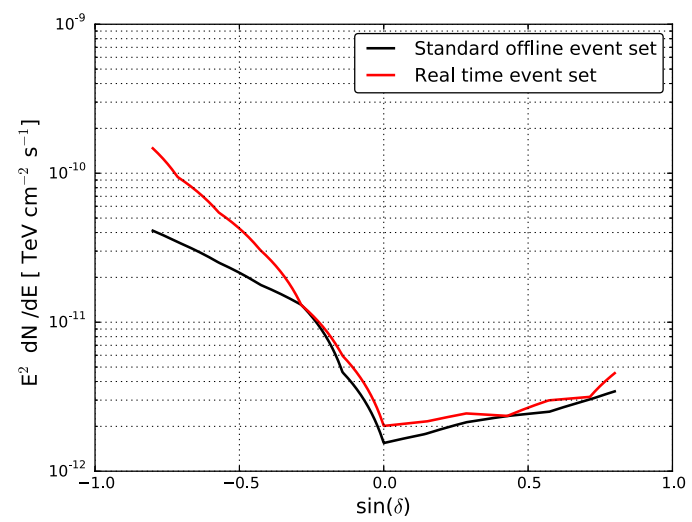

Figure 1. Sensitivity comparison for online and offline event selections as a function of the declination $\delta$. The offline selection is performed after transferring the data to the North, while the online selection runs within the limited resources at the South Pole.

In case the significance passes a threshold and permitting visibility (which may be impaired due to the moon or the zenith angle of the source w.r.t. the telescope up to 24 hours after the alert) the alert is forwarded to the MAGIC and/or VERITAS telescope.

Since the inauguration of the program in March 2012, the significance threshold was passed 14 times. Three alerts have been sent to MAGIC and five to VERITAS. One alert has been followed up by each telescope. In both cases, no significant gamma-ray emission was observed.

In Fig. 1 the sensitivity of the likelihood analysis is compared to that of the offline point source analysis. For the majority of the sky, the sensitivity of both analyses is comparable, with the offline event selection being slightly more refined to the availability of more time-consuming reconstructions.

\subsection{Optical and X-ray follow-up}

The optical and X-ray follow-up programs share the same event selection and alert generation technique. Similar to the GFU, the selection of track-like muon events is using a multivariate classifier, but this program is limited to the northern hemisphere and optimized equally for $E^{-1}, E^{-2}$ and $E^{-3}$ spectra (whereas the GFU is optimized for an $E^{-2}$ spectrum). As the majority of the events is still of atmospheric origin (muons and neutrinos), a further cut is placed allowing only cluster of neutrinos to pass that are separated by less than 3.5 degrees and arrive within 100 seconds of each other. The very short timescale of the clustering is targeted at observing neutrinos from gamma-ray bursts (GRBs) and supernovae.

Clusters of two events matching these criteria occur one or twice per week, but a cluster of three or more events passing the cut has never been observed. The alert rate is reduced further by additional cuts involving the angular separation, the reconstruction quality, the follow-up telescope's field of view and the temporal distance of the events, yielding a rate of nine alerts per year to the Palomar Transient Factory (PTF [7]) and seven alerts per year to the SWIFT X-Ray Telescope [8].

On March 30th, 2012, the most significant alert from the OFU program was sent to PTF. Two neutrinos were recorded within 1.7 seconds with a spatial separation of roughly one degree. In the follow-up observation performed by PTF ten days later, the previously unknown core-collapse supernova PTF12csy was discovered 0.14 degrees away from the mean neutrino direction. Figure 2 shows the location of the neutrinos and the supernova, as well as the result of the follow-up observations. Archival searches of the Pan-STARRS1 survey concluded the age of PTF12csy to be at least 169 days at the time of the alert. Considering the probability to find a random core-collapse supernova within 

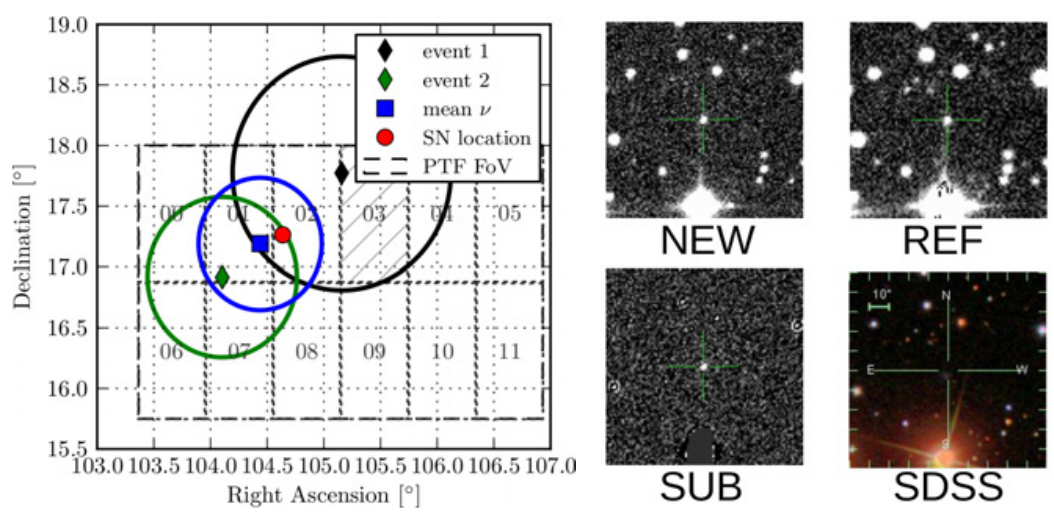

Figure 2. Left: Location of the two neutrinos that triggered the alert to PTF. The field of view of the PTF CCDs is given by the gray boxes (CCD 03 was broken). Right: Result of the PTF observation after the IceCube trigger and earlier image from the SDSS.

the neutrino error circle and the probability of the alert itself, a combined significance of $2.2 \sigma$ cannot exclude a chance detection and the neutrinos are considered to be unrelated to the supernova [9].

\section{Summary and outlook}

The IceCube experiment is able to reconstruct and transmit events, upon which alerts can be generated almost immediately. In the past, the gamma-ray and optical/X-ray follow-up programs have demonstrated reliably that these alerts can successfully trigger multi-messenger observations. In the future, a more refined event selection and updated reconstruction algorithms at the South Pole will improve the sensitivity further. Real-time selections of other event classes than through-going muons (such as starting events) will provide new follow-up opportunities.

\section{References}

[1] T.K. Gaisser, T. Stanev, Astropart. Phys. 39-40, 120 (2012), 1202.0310

[2] M.G. Aartsen et al. (IceCube), Astrophys. J. 809(1), 98 (2015), 1507.03991

[3] M.G. Aartsen et al. (IceCube), Astrophys. J. 796(2), 109 (2014), 1406. 6757

[4] A.M. Atoyan, C.D. Dermer, New Astron. Rev. 48, 381 (2004), astro-ph/0402646

[5] A. Achterberg et al. (IceCube), Astropart. Phys. 26, 155 (2006), astro-ph/0604450

[6] M. Ackermann et al., Astrophys. J. 743, 171 (2011), 1108.1420

[7] N.M. Law et al., PASP 121, 1395 (2009), 0906.5350

[8] N. Gehrels et al. (Swift Science), Astrophys. J. 611, 1005 (2004), [Erratum: Astrophys. J. 621, 558 (2005)], astro-ph/0405233

[9] M.G. Aartsen et al. (IceCube, Pan-STARRS1 Science Consortium, Swift, PTF), Astrophys. J. 811(1), 52 (2015), 1506.03115 